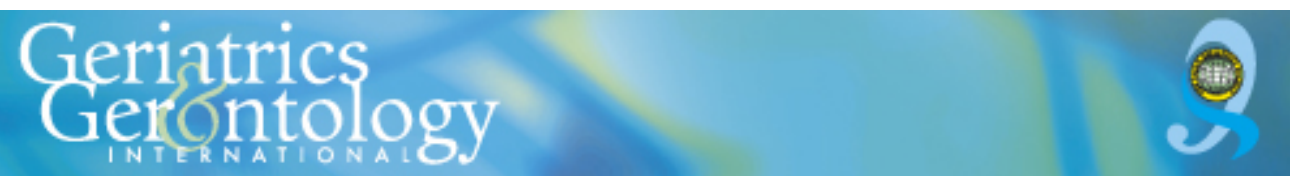

\title{
Similarities and differences in health-related behavior clustering among older adults in east and west: A latent class analysis of global aging cohorts
}

\begin{tabular}{|c|c|}
\hline Journal: & Geriatrics \& Gerontology International \\
\hline Manuscript ID & GGI-0043-2019.R1 \\
\hline Manuscript Type: & Original Article \\
\hline $\begin{array}{l}\text { Date Submitted by the } \\
\text { Author: }\end{array}$ & 15-May-2019 \\
\hline Complete List of Authors: & $\begin{array}{l}\text { Liao, Jing; Sun Yat-Sen University, School of Public Health, Department } \\
\text { of Medical Statistics; Sun Yat-Sen University, Sun Yat-sen Global Health } \\
\text { Instittute } \\
\text { Mawditt, Claire; NHS Foundation Trust } \\
\text { Scholes, Shaun; University College London, Department of Epidemiology } \\
\text { and Public Health } \\
\text { Lu, Wentian; University College London Research Department of } \\
\text { Epidemiology and Public Health } \\
\text { Umeda, Maki; University of Hyogo, Global Health Nursing, Research } \\
\text { Institute of Nursing Care for People and Community Global Health } \\
\text { Nursing, Research Institute of Nursing Care for People and Community } \\
\text { Muniz Terrera, Graciela; University of Edinburgh, Centre for Dementia } \\
\text { Prevention } \\
\text { Hao, Yuantao; Sun Yat-Sen University, School of Public Health } \\
\text { Department of Medical statistics } \\
\text { Mejía, Shannon; University of Illinois at Urbana-Champaign Mathematics } \\
\text { Library, Department of Kinesiology \& Community Health College of } \\
\text { Applied Health Sciences, }\end{array}$ \\
\hline Keywords: & Epidemiology < Socio-Medical Science, Public Health < Basic Science \\
\hline Optional Keywords: & $\begin{array}{l}\text { clustering, health-related behaviors, latent class analysis, multiple risk } \\
\text { factors }\end{array}$ \\
\hline
\end{tabular}

\section{SCHOLARONE ${ }^{\text {m }}$ Manuscripts}




\section{Similarities and differences in health-related behavior clustering among older adults in east and west: A latent class analysis of global aging cohorts}

Running title: Behavior cluster of east \& west elderly

Jing Liao, ${ }^{1}$ Claire Mawditt,,${ }^{2}$ Shaun Scholes, ${ }^{3}$ Wentian Lu, ${ }^{3}$ Maki Umeda, ${ }^{4}$ Graciela Muniz Terrera,,${ }^{5}$ Yuantao Hao, ${ }^{1 *}$ Shannon Mejía ${ }^{6}$

1. Department of Medical Statistics and Epidemiology | Sun Yat-sen University Global Health Institute, School of Public Health | Institute of State Governance, Sun Yat-sen University, Guangzhou, China

2. Derby Teaching Hospitals NHS Foundation Trust, London, United Kingdom,

3. Department of Epidemiology and Public Health, University College London, London, United Kingdom

4. Global Health Nursing, Research Institute of Nursing Care for People and Community, University of Hyogo, Hyogo, Japan

5. Centre for Dementia Prevention, University of Edinburgh, Edinburgh, United Kingdom

${ }^{6}$. Department of Kinesiology \& Community Health College of Applied Health Sciences, University of Illinois at Urbana-Champaign, Champaign, United States

* Corresponding author: Yuantao Hao, haoyt@mail.sysu.edu.cn, Department of Medical Statistics and Epidemiology, School of Public Health, Sun Yat-sen University, No.74 Zhongshan 2nd Road, Guangzhou, China, 51008

\section{Word count:}

Text: $2867-2904$

Figures: 3 Tables: 2 


\begin{abstract}
Aim: To quantify variations in health-related behaviors (HRBs) clustering of older adults in western and eastern countries.

Methods: Using six aging cohorts from the US, England, Europe, Japan, Korea and China, latent class analysis was applied to access the clustering of smoking, alcohol consumption, physical activity, and social activity.

Results: A total of 104,552 participants (55\% women) aged $\geq 50$ years in 2010 were included. Despite different number of clusters identified, three consistent cluster profiles emerged: 'Multiple-HRBs' (ex/never-smoking, moderate drinking, frequent physical- and social- activity); 'Inactives' (socially and physically inactive without other risk behaviors); and '(ex-)Smokers with Risk Behaviors'. Gender- and cohort- variations showed. For men in western cohorts, 'Multiple-HRBs' was the predominant cluster, whereas their Asian counterparts were more likely to be members of the 'Smokers with risk behavior' and 'Inactives' clusters. Most women, particularly those in Asian cohorts, were never-smokers and non-drinkers, and most of them belonged to the socially 'Inactives' cluster.
\end{abstract}

Conclusions: We provide a person-centered understanding on HRB clustering of older adults over selected countries by gender, informing tailored health promotion for the target population.

Keywords: clustering; health-related behaviors; latent class analysis; multiple risk factors. 


\section{Introduction}

Extending the lifespan of effective functioning and independence is a public health priority worldwide. Modifiable health-related behaviors (HRBs) are key contributors to chronic diseases and early mortality even in older age; ${ }^{1}$ insofar as by maintaining a healthy lifestyle, the processes of frailty, disability, and dementia can be delayed. $^{2}$

Despite cultural and socio-demographic differences, healthy lifestyle recommendations are similar across countries, namely, non-smoking, non-excessive alcohol consumption, and regular physical exercise. $^{2}$ International epidemiological studies have demonstrated that HRBs do not co-occur within individuals by chance, but rather cluster, ${ }^{3}$ where a given combination of HRBs is more prevalent than expected if they were independent. ${ }^{4}$ Smoking, alcohol and physical activity were the most frequently studied HRBs. The clustering of smoking with alcohol, and the absence or presence of all HRBs have been identified fairly consistently in Europe, ${ }^{5}$ the United Kingdom (UK) ${ }^{6}$ and the United States (US) ${ }^{3}$, but evidence in Asian countries is scarce.7,8

Research on HRB clustering in older-age populations has, however, been limited. Only eight out of the 56 studies on HRB clustering (till 2014) reviewed by Noble and colleges ${ }^{3}$ focused on adults aged 50 years and above, besides a few recent updates..$^{5,9}$ Some of these studies suggest that older adults tend to adopt more positive HRBs (e.g. less likely to smoke, moderate alcohol consumption) than their younger counterpart; ${ }^{9-11}$ whereas other studies have found that older age is associated with more negative HRBs, ${ }^{7}$ ${ }^{8}$ reflecting heterogeneous lifestyle. Age-related functional declines may also result in specific HRB clustering in older age, driven by less frequent physical activity ${ }^{7,10,11}$ and social participation. ${ }^{12}$ Only one study ${ }^{13}$ considered social interaction and church attendance. However, as it was on religious involvement only, the HRB clusters identified may not apply to other non-religious populations.

Furthermore, substantial differences in methodologies across studies make it difficult to draw consensus about HRB clustering. ${ }^{3}$ Previous studies used different selection and measurement of HRBs, with some studies likely to be limited by selection-bias. ${ }^{3}$ A number of studies calculated the observed/expected prevalence ratio to study the strength of HRB clustering, ${ }^{7-9}$ while others used datareduction techniques. The utilization of more advanced techniques such as latent class analysis (LCA) allows the identification of underlying HRB clustering in a person-centered manner, and provides more analytic options other than dichotomized measures. ${ }^{4}$ 
Our study aimed to quantify variations in HRB clustering across an expanded representation of western and eastern countries, by applying the same statistical method LCA to six population-representative aging cohorts with comparable HRB measures from the US, England, Europe, Japan, Korea, and China.

\section{Methods}

\section{Study population}

Individual participant data from six ageing cohorts were used: the Health and Retirement Study (HRS); ${ }^{14}$ the English Longitudinal Study of Ageing (ELSA); ${ }^{15}$ the Survey of Health Ageing and Retirement in Europe (SHARE); ${ }^{16}$ the Japanese Longitudinal Study of Ageing (JSATR);17 the Korean Longitudinal Study of Aging (KLoSA) ${ }^{18}$ and the China Health and Retirement Longitudinal Study (CHARLS). ${ }^{19}$ Variables were harmonized in the gateway of global aging data to facilitate cross national comparisons. Our study included participants aged $\geq 50$ years in 2010 with at least one HRB measure. Considering lifestyle variations, SHARE was regrouped as Northern Europe (Demark and Sweden), Western Europe (Netherlands, Germany, Belgium, Switzerland and France), Southern Europe (Italy, Spain and Portugal), and Eastern/Central Europe (the Czech Republic, Austria, Slovenia, Poland and Hungary), based on traditions, behavioral patterns and the Active Aging Index. ${ }^{20}$ Above cohorts had obtained ethical approval from the relevant committees; all participants signed informed consent.

\section{Health-related behavior measures}

In accordance with the literature ${ }^{1,4}$ and available measures in the selected cohorts, four HRBs were chosen (Harmonization details see Table S1). Smoking was categorized as non-smoker, ex-smoker, or current smoker, deriving from participant's smoking history and current smoking behavior. Drinking was grouped as 'non-drinker', '0-2 drinks/day' or ' $>2$ drinks/day' based on the frequency and quantity of alcohol consumption, Physical activity was divided as 'non-weekly exercise' and 'weekly exercise' (moderate or vigorous physical activity at least once per week). It was constructed to reflect the evidence-based physical $\underline{\text { activity recommendations for older adults by } \mathrm{WHO}^{21}}$; and to maximize the comparability of survey-specific questions, as KLoSA and JSTAR only asked participants weekly 'work out' or 'exercise' frequency without specific activity type (e.g. vigorous or moderate). Social activity (i.e. conducting volunteer or charity work, attending an educational or training course, being a member of a sport, social, or other club, or attending non-/religious meetings or activities) was quantified as 'frequent' if participants reported doing these activities no less than once per week, or 'infrequent' if participating less frequently or not at all. 


\section{Statistical analysis}

Age-standardized prevalence of HRBs were calculated by the direct method, with the weighted population across cohorts taken as the reference for standardization. Using $\mathrm{LCA}^{22}, \mathrm{w} \underline{\mathrm{W}} \mathrm{e}$ examined cohort differences in HRB clustering in two ways: cluster patterns (i.e. number of clusters and combination of HRBs) and cluster membership (i.e. proportion of the cohort belonging to each cluster), $\underline{\text { using LCA }^{22}}$. Briefly, LCA models use respondents' responses across the target observed variables (i.e. item response probability) to identify latent classes of response patterns in the data. Our interest is on the number and characterization of classes within each cohort. Thus, we focus on the item-response probabilities that an HRB is represented within a class. Analysis was conducted in men and women separately, given substantial gender differences in HRB clustering. ${ }^{3,8}$ To select an appropriate number of HRB clusters in each cohort, several fit indices were considered, log-likelihood, adjusted Bayesian Information criterion (aBIC), entropy and the Lo-Mendell Rubin likelihood ratio test (LMR). ${ }^{22,23}$ These model indices have been identified as reliable indicators to distinguish a sub-population mixture distribution from a homogenous non-normal distribution. ${ }^{23}$ We placed more emphasis on the aBIC as it balances model fit with parsimony ${ }^{22}$ and has been found to perform well in large samples. ${ }^{23}$ We also considered cluster size being no less than 5\% and cluster interpretability. ${ }^{24}$ Missing data (14\%) were handled with the Full Information Maximum Likelihood (FIML) procedure. ${ }^{25}$ Sensitivity analyses with only complete cases $(\mathrm{N}=90,239)$ were conducted to compare findings of FIML models. Age-standardized HRB prevalence were estimated using Stata Version 14.0 and LCA were estimated by Mplus Version 7.4. ${ }^{26}$ We used radar charts to aid visual interpretation of the identified clusters. With the radial grid like structure, these charts display the item-response probabilities of HRBs for a given latent cluster on its axis.

\section{Results}

\section{Prevalence of HRBs across cohorts by gender}

The analysis included 104,552 participants $(55 \%$ female) aged $\geq 50$ years of the six cohorts (Participants demographic characteristics of each cohort were presented in Table S2). Table 1 shows age-standardized prevalence of HRBs by gender and by cohort. For men, many of the cohorts consisted of ex-smokers or non-smokers, moderate drinkers, who were physically active but socially inactive. Different HRB prevalence was found in CHARLS (i.e. 57\% current smokers and 47\% social active), and KLoSA (i.e. 55\% 
heavy drinkers and $62 \%$ physical inactive). Women from most of the cohorts were never smokers, and the majority of the three Asian cohorts were non-drinkers. Over half of women participated in weekly physical activity except for those in Korea (32\%). Apart from women in Northern Europe (51\%) and China (45\%), most women were not frequently engaged in social activities.

\section{Cluster patterns}

Model fit indices for all LCA models are presented in Table $\$ 2 \underline{\mathrm{S} 3}$ (men) and $\$ 3$ S4 (women). Although a three-class solution was the most common choice for the number of clusters, a two-class solution was suggested by model fit indices for men of ELSA, Southern-EU of SHARE, KLoSA and CHARLS, and for women of Western-EU of SHARE and JSTAR (Table 2). A two-cluster solution was also suggested for women in CHARLS, but the proportion of women belonging to the second cluster was small $(6 \%)$ and differed from the other cluster only in its higher probability of moderate alcohol consumption. It was thus decided to maintain the one-cluster solution for women in the CHARLS cohort.

Despite differences in the number of latent clusters obtained, three largely consistent HRB clustering emerged across the cohorts. Based on the response probabilities of the four HRBs within each HRB clustering (Figure $1 \&$ 2), we cautiously labelled these three clusters to aid interpretability, whilst acknowledging heterogeneity in HRB clustering across the cohorts.

Cluster 1, labelled 'Multiple-HRBs', was characterized by more positive HRBs than the other two clusters. Individuals in this cluster were likely to be ex-smokers (for men) or never smokers (for women), moderate drinkers (or non-drinkers for Asian women), and engage in frequent physical and social activities.

Cluster 2, labelled 'Inactives', was notably distinguished from the other clusters by infrequent socialand physical- activities combined with the absence of other risk behaviors. Most individuals with this behavior pattern were ex- or non-smokers, and were moderate- or non-drinkers particularly for women.

Cluster 3, labelled '(ex)Smokers with Risk Behaviors', was characterized by a higher probability of being current smokers (for men) or ex-smokers (for women). Other risk behaviors that accompanied smoking varied across the cohorts. Smoking cooccurred with high alcohol consumption amongst men of HRS, SHARE and KloSA, and amongst women of KLoSA. The risk factor of infrequent social activity was broadly present in this cluster across cohorts, except for men from CHARLS. Additionally, for KLoSA participants, a profile of non-weekly exercise was present. 


\section{Cluster membership}

As shown in Figure 3, for both men and women, the proportion of participants belonging to HRB cluster differed across cohorts. For men, over half of the ELSA, HRS and SHARE participants were classified as belonging to the 'Multiple-HRBs' cluster (77\%, 63\%, and 72\% respectively), whereas JSTAR and CHARLS were dominated by 'Smokers with Risk Behaviors' (53\% and 60\% respectively), and 60\% of KLoSA were in the 'Inactives' clusters.

For women, only western-EU and CHARLS had most participants belonging to the 'Multiple-HRBs' cluster (83\% and 100\% respectively). All three patterns were well-represented in HRS and in the easternEU of SHARE. Around half of ELSA, northern- and southern-EU participants were '(ex)Smokers with Risk Behaviors', with the secondary cluster of 'Multiple-HRBs' for northern EU (43\%) and ELSA (33\%). KLoSA was dominated by both 'Multiple-HRBs' (51\%) and 'Inactives' (41\%) clusters. JSTAR was predominately comprised of 'Inactives' (83\%). These findings, using FIML to accommodate missing data, were confirmed by our sensitivity analyses.

\section{Discussion}

We applied LCA to capture similarities and differences in HRB clustering of smoking, alcohol consumption, physical activity, and social activity across six highly-comparable aging cohorts of community-dwelling persons aged 50 years and older. Our results showed three largely consistent HRB clustering: 'MultipleHRBs' characterized by multiple positive HRBs; 'Inactives' cluster distinguished by infrequent social- and physical-activities but no other risk behaviors; '(ex)Smokers with Risk Behaviors' cluster with current smoking in men and with ex-smoking in women, coupled by excessive drinking, and being socially- or physically-inactive. Gender- and cohort-specific variations in these cluster patterns and differences in the proportion belonging to each cluster were also revealed.

Our study contributes to the literature by directly demonstrating the general similarities alongside variations in HRB clustering across western and eastern aging cohorts. The three common cluster patterns found here largely match the HRB structural compositions identified in recent reviews, particularly regarding the clustering of multiple positive $\mathrm{HRBs},{ }^{3,5}$ and the clustering of smoking and heavy alcohol consumption., 6 Our findings showed gender- and cohort-specific patterns of smoking and alcohol consumption. For men, ex-smokers and moderate-drinkers were included in the 'Multiple-HRBs' cluster, while current smoking and excessive drinking were the main risk behaviors for the '(ex)Smokers with risk 
behavior' cluster. For women, most of them were never-smokers in the 'Multiple-HRBs' cluster and were ex-smokers in the '(ex)Smokers with Risky Behaviors' cluster, with women of the Asian cohorts being most likely to abstain from alcohol. The variation in gendered cluster patterns identified here are consistent with literature. For example, research has found a much lower prevalence of current smoking and alcohol consumption in elderly Asian women than men, $7,8,11,17$ resulting from traditional social norm and cultural constraints. In contrast, there is a convergence in alcohol consumption between Western women and men, mainly due to increased consumption amongst women. ${ }^{27}$

By incorporating the social engagement component, a key determinant of active aging, ${ }^{28}$ our study provides a more comprehensive description of HRB clustering in older age. We found that the agestandardized prevalence of frequent social activity was low in most cohorts, except for Northern EU of SHARE and for CHARLS. Despite the limitations of the social activity items within individual survey, our findings highlight the prevalently low levels of social engagements among older adults. However, the frequency of social engagement has rarely been explored in previous HRB clustering studies and less is known about its association with other HRBs. Our results show that the 'Inactives' cluster was characterized by socially inactive participants, whose estimated probability of participating in physical activity at least once a week were lower than the other two clusters; yet they neither smoked nor drunk alcohol excessively. Comparable clusters characterized by insufficient physical activity with no other risk behaviors have been found in German ${ }^{5,10}$ and Taiwanese ${ }^{11}$ middle- to older- aged samples, without reference to participants' social activity levels. Current health promotion programs mainly focus on smoking, drinking, and physical activity ${ }^{2}$ which appears less relevant to this group. Our findings indicate the need for greater emphasis on building pathways to facilitate social engagement in older adulthood.

The varied prevalence of cluster membership across the cohorts is also noteworthy. For men in each Western cohort, 'Multiple-HRBs' was the predominant cluster, whereas their Asian counterparts were more likely to be members of the '(ex)Smokers with Risk Behavior' and 'Inactives' clusters. Socio-cultural tolerance for smoking may help to explain the low smoking cessation rate amongst men from the Japanese and Chinese cohorts. In China, smoking is regarded as necessary for socializing among men especially amongst older generations with poor health literacy. ${ }^{29}$ This is in keeping with our findings that smoking was associated with frequent social activity in the CHARLS cohort. In both countries, smoking is permitted in public places and cigarette taxation is low, partially due to strong opposition from the tobacco and 
catering service industries..$^{29,30}$

Corresponding to our findings on the largest 'Inactives' cluster in KLoSA, a low rate of adherence to healthy lifestyles, particularly with infrequent physical activity in older Korean men, has been reported by Lee and colleagues. ${ }^{8}$ The authors suggested that the lack of evidence-based programs and co-ordination between governmental agencies for physical activity promotion should take the blame. The 'Inactives' cluster was also predominant in women of KLoSA, southern EU and JSTAR. The strikingly-high proportion of socially inactive women in the Japanese cohort, who otherwise had no other risk behaviors, may be explained by the persistent gender roles in Japan. Women are expected to undertake the majority of domestic duties, leading to constraints and a reluctance in spending time for engaging with social networks beyond their immediate family. ${ }^{31}$

The strength of our study lies in its utilization of six comparable population-representative aging cohorts, which provides a unique opportunity to conduct a multinational study of HRB clustering on a scale that has not been achieved before. Our study lends important insights into the similarities and differences in the clustering of HRBs evident amongst middle- and older-aged adults across sociocultural heterogeneous populations.

Nevertheless, the interpretation of these findings requires caution. First, this study relies on selfreported HRB measures. Participants may respond to these questions in line with social desirability. We suspected that uniformed bias in underestimation of health damaging behaviors and the over-reporting of health promoting behaviors may exist across the cohorts. Second, it is acknowledged that the HRB measures were not identical across the cohorts, despite considerable efforts made in variable harmonization. Confined to the measures available, our study was unable to make more fine-graded categories for some harmonized measures; or include more lifestyle factors, such as nutrition, which was absent in most of the study cohorts. Further replication of our study using other datasets with more detailed HRB measures would be advantageous.

Third, the current study only examined HRB clustering by gender and cohort, which may also be influenced by socioeconomic status. Despite that the application of survey weights may have accounted for some of the differences, it will be important for future research to tease out socioeconomic variations from cultural mechanisms. Last,Fourth, the current study only used HRB measures assessed at one time point. This overlooks cumulative exposure to these factors and so cannot depict changes in HRBs with age. 
Although within-person change in HRBs may not be substantial in older adulthood, examining behavioral patterns over multiple waves would advance our knowledge of how divergent longitudinal patterns of HRBs unfold in the transition from middle to older age. Last, as the main purpose of our current study was to empirically derive and compare HRB clustering patterns across western and eastern cohorts, the extent to which these complex webs of HRB patterns would be associated with distal health outcomes has not yet been explored. Building upon these identified HRB clusters, our subsequent studies will systematically investigate how participants with different HRB profiles would age physically and mentally against the global ageing context.

In conclusion, we provide a fine-grained analysis on HRB clustering across six aging cohorts in east and west. Our findings suggest a large degree of consistency in HRB clustering across western and eastern cohorts, but with gender- and cohort- variations in cluster pattern and membership. A person-centered understanding of the combination and distribution of multiple HRBs may facilitate identifying subpopulations most at risk and informing the design of gender- and context-specific health promotion programs.

\section{Acknowledgements}

This study used information from the RAND HRS, the Harmonized ELSA (Version E), Harmonized SHARE (Version C.3), Harmonized JSTAR (Version B), Harmonized KLoSA (Version B) and Harmonized CHARLS (Version B.4) datasets. The development of these harmonized datasets was funded by the National Institute on Aging (R01 AG030153, RC2 AG036619, 1R03AG043052). For more information, please refer to www.g2aging.org. JL receive funds from the Guangdong Natural Science Foundation (2017A030310346, 2018A0303130046) and Sun Yat-sen Scientific Research Fund for Early Career Researchers (17ykpy15). The funders had no role in study design, data analysis and interpretation or writing of the manuscript.

\section{Disclosure statement}

There is no conflict interest to be claimed.

\section{References}

[1] Rizzuto D, Fratiglioni L. Lifestyle factors related to mortality and survival: a mini-review. Gerontology 2014; 60: 327-35.

[2] NICE. Dementia, disability and frailty in later life - mid-life approaches to delay or prevent onset. . 2015.

[3] Noble N, Paul C, Turon H, Oldmeadow C. Which modifiable health risk behaviours are related? A systematic review of the clustering of Smoking, Nutrition, Alcohol and Physical activity ('SNAP') health risk factors. Preventive medicine 2015; 81: 16-41.

[4] McAloney K, Graham H, Law C, Platt L. A scoping review of statistical approaches to the analysis of multiple health-related behaviours. Preventive medicine 2013; 56: 365-71.

[5] Jentsch F, Allen J, Fuchs J, von der Lippe E. Typical patterns of modifiable health risk factors (MHRFs) 
in elderly women in Germany: results from the cross-sectional German Health Update (GEDA) study, 2009 and 2010. BMC women's health 2017; 17: 23.

[6] Meader N, King K, Moe-Byrne T, et al. A systematic review on the clustering and co-occurrence of multiple risk behaviours. BMC public health 2016; 16: 657.

[7] Chou K-L. The prevalence and clustering of four major lifestyle risk factors in Hong Kong Chinese older adults. Journal of aging and health 2008; 20: 788-803.

[8] Lee Y, Back JH, Kim J, Byeon H, Kim S, Ryu M. Clustering of multiple healthy lifestyles among older Korean adults living in the community. Geriatrics \& gerontology international 2012; 12: 515-23.

[9] Morris LJ, D'Este C, Sargent-Cox K, Anstey KJ. Concurrent lifestyle risk factors: Clusters and determinants in an Australian sample. Preventive medicine 2016; 84: 1-5.

[10] Schneider S, Huy C, Schuessler M, Diehl K, Schwarz S. Optimising lifestyle interventions: identification of health behaviour patterns by cluster analysis in a German 50+ survey. European journal of public health 2009; 19: 271-77.

[11] Hsu H-C, Luh D-L, Chang W-C, Pan L-Y. Joint trajectories of multiple health-related behaviors among the elderly. International journal of public health 2013; 58: 109-20.

[12] Pinto JM, Neri AL, Pinto JM, Neri AL. Trajectories of social participation in old age: a systematic literature review. Revbrasgeriatrgerontol 2017; 20: 259-72.

[13] Norton MC, Dew J, Smith H, et al. Lifestyle behavior pattern is associated with different levels of risk for incident dementia and Alzheimer's disease: the Cache County study. Journal of the American Geriatrics Society 2012; 60: 405-12.

[14] Sonnega A, Faul JD, Ofstedal MB, Langa KM, Phillips JW, Weir DR. Cohort Profile: the Health and Retirement Study (HRS). Int J Epidemiol 2014; 43: 576-85.

[15] Steptoe A, Breeze E, Banks J, Nazroo J. Cohort profile: the English longitudinal study of ageing. International Journal of Epidemiology 2012; 42: 1640-48.

[16] Börsch-Supan A, Brandt M, Hunkler C, et al. Data resource profile: the Survey of Health, Ageing and Retirement in Europe (SHARE). International Journal of Epidemiology 2013: dyt088.

[17] Ichimura H, Shimizutani S, H. H. JSTAR First Results 2009 Report. Research Institute of Economy, Trade and Industry (RIETI) 2009.

[18] Park Y, Freedman AN, Gail MH, et al. Validation of a colorectal cancer risk prediction model among white patients age 50 years and older. J Clin Oncol 2009; 27: 694-8.

[19] Zhao Y, Hu Y, Smith JP, Strauss J, Yang G. Cohort profile: The China health and retirement longitudinal study (CHARLS). International journal of epidemiology 2012; 43: 61-68.

[20] Zaidi A, Gasior K, Hofmarcher MM, et al. Active ageing index 2012 concept, methodology and final results. 2013.

[21] World health organization. Global recommendations on physical activity for health Switzerland: WHO, 2010.

[22] Collins LM, Lanza ST. Latent Class and Latent Transition Analysis: With Applications in the Social, Behavioral, and Health Science: Wiley, 2010.

[23] Nylund, Karen L, Asparouhov, Tihomir, Muthén, Bengt O. Deciding on the number of classes in latent class analysis and growth mixture modeling: A Monte Carlo simulation study. Structural Equation Modeling A Multidisciplinary Journal 2007; 14: 535-69.

[24] Wang J, Wang X. Structural equation modeling: Applications using Mplus: John Wiley \& Sons, 2012. [25] Enders CK, Bandalos DL. The relative performance of full information maximum likelihood estimation for missing data in structural equation models. Structural Equation Modeling 2001; 8: 430-57. [26] Muthen M. Mplus: Version 7. Los Angeles, CA: Muthen \& Muthen, 2014.

[27] Mawditt C, Sacker A, Britton A, Kelly Y, Cable N. The clustering of health-related behaviours in a British population sample: Testing for cohort differences. Preventive medicine 2016; 88: 95-107.

[28] World Health Organization. Active Ageing: A Policy Framework. Geneva, Switzerland: WHO, 2002. [29] Hu T-W, Lee AH, Mao Z. WHO Framework Convention on Tobacco Control in China: barriers, challenges and recommendations. Economics of Tobacco Control in China: From Policy Research to Practice: World Scientific, 2016; 247-63.

[30] Tsugane S. Alcohol, smoking, and obesity epidemiology in Japan. Journal of Gastroenterology \& Hepatology 2012; 27: 121-26.

[31] Gender Equality Bureau Cabinet Office. Women and men in Japan 2018. Government of Japan, 2018. 


\section{Figure Legends}

Figure 1. Radars charts of item-response probabilities of health-related behavior clustering for three latent clusters by cohort in men. 'Multiple-HRBs': ex-smoking, moderate drinking, frequent physical and social activity (Black line); 'Inactives': socially and physically inactive without other risk behaviors (Grey line); and '(ex)Smokers with Risk Behaviors' (Black dash line). Data from participants aged $\geq 50$ years in 2010 Health and Retirement Study (HRS), English Longitudinal Study of Ageing (ELSA), the Survey of Health Ageing and Retirement in Europe (SHARE), Japanese longitudinal study of Ageing (JSTAR), Korean Longitudinal Study of Aging (KLoSA) and China Health and Retirement Longitudinal Study (CHARLS).

Figure 2. Radars charts of item-response probabilities of health-related behavior clustering for three latent clusters by cohort in women. 'Multiple-HRBs': never-smoking, non/moderate drinking, frequent physical and social activity (Black line); 'Inactives': socially and physically inactive without other risk behaviors (Grey line); and '(ex)Smokers with Risk Behaviors' (Black dash line). Data from participants aged $\geq 50$ years in 2010 Health and Retirement Study (HRS), English Longitudinal Study of Ageing (ELSA), the Survey of Health Ageing and Retirement in Europe (SHARE), Japanese longitudinal study of Ageing (JSTAR), Korean Longitudinal Study of Aging (KLoSA) and China Health and Retirement Longitudinal Study (CHARLS).

Figure 3. The proportions of health-related behavior cluster membership by gender and cohort. Data from participants aged $\geq 50$ years in 2010 Health and Retirement Study (HRS), English Longitudinal Study of Ageing (ELSA), the Survey of Health Ageing and Retirement in Europe (SHARE), Japanese longitudinal study of Ageing (JSTAR), Korean Longitudinal Study of Aging (KLoSA) and China Health and Retirement Longitudinal Study (CHARLS). 


\section{Supporting Information}

Table S1. Cohort-specific health-related behavior questionnaire item and harmonizing strategy.

Table S2. Sociodemographic characteristics of study cohorts.

Table S2 $\underline{S}$. Goodness of fit indices for latent profile analysis models, by cohort for men.

Table S3 $\underline{\text { S4}}$. Goodness of fit indices for latent profile analysis models, by cohort for women. 
Table 1. Age-standardized health-related behavior characteristics of the analytical sample by gender and cohort. Data from participants aged $\geq 50$ years in 2010 Health and Retirement Study (HRS), English Longitudinal Study of Ageing (ELSA), the Survey of Health Ageing and Retirement in Europe (SHARE), Japanese longitudinal study of Ageing (JSTAR), Korean Longitudinal Study of Aging (KLoSA) and China Health and Retirement Longitudinal Study (CHARLS).†

\begin{tabular}{|c|c|c|c|c|c|c|c|c|c|c|c|c|c|c|c|c|c|c|c|}
\hline \multicolumn{2}{|l|}{10} & \multicolumn{9}{|c|}{ Men } & \multicolumn{9}{|c|}{ Women } \\
\hline 12 & \multirow{2}{*}{$\begin{array}{l}\text { Cohort } \\
\text { Country }\end{array}$} & \multirow{2}{*}{$\begin{array}{c}\text { ELSA } \\
\text { England }\end{array}$} & \multirow{2}{*}{$\begin{array}{c}\text { HRS } \\
\text { US }\end{array}$} & \multirow[b]{2}{*}{$\mathbf{N}-\mathbf{E U}$} & \multicolumn{2}{|c|}{ SHARE } & \multirow[b]{2}{*}{$\mathbf{E}-\mathbf{E} \mathbf{U}$} & \multirow{2}{*}{$\begin{array}{l}\text { JSTAR } \\
\text { Japan }\end{array}$} & \multirow{2}{*}{$\begin{array}{c}\text { KLoSA } \\
\text { Korea }\end{array}$} & \multirow{2}{*}{$\begin{array}{c}\text { CHARLS } \\
\text { China }\end{array}$} & \multirow{2}{*}{$\begin{array}{l}\text { ELSA } \\
\text { England }\end{array}$} & \multirow{2}{*}{$\begin{array}{c}\text { HRS } \\
\text { US }\end{array}$} & \multicolumn{4}{|c|}{ SHARE } & \multirow{2}{*}{$\begin{array}{c}\text { JSTAR } \\
\text { Japan }\end{array}$} & \multicolumn{2}{|c|}{ KLoSACHARLS } \\
\hline $\begin{array}{l}13 \\
14\end{array}$ & & & & & W-EU & S-EU & & & & & & & N-EU & W-EU & S-EU & E- EU & & Korea & China \\
\hline 15 & No. & 4,010 & 8,777 & 1,921 & 8,374 & 4,102 & 7,644 & 2,032 & 3,410 & 6,714 & 4,973 & 11,560 & 2,240 & 10,237 & 4,988 & 10,103 & 2,114 & 4,510 & 6,843 \\
\hline \multicolumn{20}{|c|}{16 Smoking status $(\%)$} \\
\hline & ver smoker & 31.4 & 35.9 & 40.1 & 36.4 & 36.7 & 32.7 & 27.8 & 61.0 & 26.7 & 42.8 & 49.4 & 43.0 & 60.8 & 70.0 & 57.7 & 81.4 & 97.2 & 90.7 \\
\hline 191 & smoker & 53.2 & 46.9 & 39.3 & 40.8 & 37.2 & 34.0 & 44.7 & 0.9 & 16.8 & 41.7 & 35.9 & 37.7 & 21.1 & 15.0 & 18.0 & 10.2 & 0.1 & 2.3 \\
\hline $\begin{array}{l}20 \\
21 \\
22\end{array}$ & $\begin{array}{l}\text { rent smoker } \\
\text { inking (\%) }\end{array}$ & 15.4 & 17.2 & 20.6 & 22.8 & 26.1 & 33.3 & 27.5 & 38.1 & 56.5 & 15.5 & 14.7 & 19.3 & 18.1 & 15.0 & 24.3 & 8.4 & 2.7 & 7.0 \\
\hline 231 & -drinker & 5.5 & 17.3 & 1.5 & 2.3 & 9.2 & 5.6 & 33.3 & 29.0 & 24.0 & 9.4 & 26.7 & 3.6 & 7.6 & 34.5 & 20.1 & 72.6 & 77.9 & 75.7 \\
\hline $24 c$ & /day & 57.4 & 65.0 & 63.3 & 71.1 & 71.8 & 65.3 & 49.7 & 15.8 & 67.5 & 74.8 & 68.2 & 79.9 & 82.0 & 60.6 & 70.7 & 21.8 & 9.6 & 23.8 \\
\hline $\begin{array}{l}25 \\
26\end{array}$ & & 37.1 & 17.7 & 35.2 & 26.6 & 19.0 & 29.1 & 17.0 & 55.2 & 8.5 & 15.8 & 5.1 & 16.5 & 10.4 & 4.9 & 9.2 & 5.7 & 12.5 & 0.6 \\
\hline \multicolumn{20}{|c|}{27 Weekly physical activity $(\%)$} \\
\hline 28 & ive & 78.6 & 75.0 & 92.5 & 88.6 & 79.7 & 77.0 & 69.3 & 37.5 & 61.0 & 74.5 & 68.5 & 91.6 & 87.2 & 73.3 & 74.7 & 83.0 & 32.1 & 54.3 \\
\hline${ }_{30}^{29} \mathrm{I}$ & ctive & 21.4 & 25.0 & 7.5 & 11.4 & 20.3 & 23.0 & 30.7 & 62.5 & 39.0 & 25.5 & 31.5 & 8.4 & 12.8 & 26.7 & 25.3 & 17.0 & 67.9 & 45.7 \\
\hline \multicolumn{20}{|c|}{$\begin{array}{l}30 \\
31\end{array}{ }^{3}$ Frequent social activity $(\%)$} \\
\hline 32 & & 38.0 & 27.7 & 42.9 & 36.0 & 22.0 & 26.4 & 20.3 & 24.1 & 45.6 & 36.7 & 29.6 & 51.2 & 38.5 & 24.3 & 29.6 & 16.2 & 32.8 & 45.0 \\
\hline $33 r$ & & 62.0 & 72.3 & 57.1 & 64.0 & 78.0 & 73.6 & 79.7 & 75.9 & 54.4 & 63.3 & 70.4 & 48.8 & 61.5 & 75.7 & 70.4 & 83.8 & 67.2 & 55.0 \\
\hline
\end{tabular}

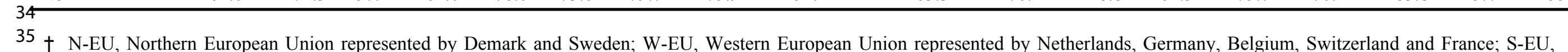


Table 2. Goodness of fit indices for chosen cluster solution by gender and cohort. Data from participants aged $\geq 50$ years in 2010 Health and Retirement Study (HRS), English Longitudinal Study of Ageing (ELSA), the Survey of Health Ageing and Retirement in Europe (SHARE), Japanese longitudinal study of Ageing (JSTAR), Korean Longitudinal Study of Aging (KLoSA) and China Health and Retirement Longitudinal Study (CHARLS).

\begin{tabular}{|c|c|c|c|c|c|c|}
\hline Cohort & $\begin{array}{l}\text { No. of } \\
\text { Clusters }\end{array}$ & Loglikelihood & aBIC & Entropy & $\begin{array}{c}\text { LMR-LRT } \\
\text { p-value }\end{array}$ & $\begin{array}{c}\text { Smallest Size } \\
\underline{\mathbf{N}}(\%)\end{array}$ \\
\hline & & & Men & & & \\
\hline ELSA-UK & 2 & -10956.50 & 21979.55 & $\underline{0.72}$ & $<0.001$ & $925 \underline{(23 \%)}$ \\
\hline HRS-US & 3 & -26381.36 & 52944.32 & $\underline{0.89}$ & $<0.0001$ & $1414(16 \%)$ \\
\hline \multicolumn{7}{|l|}{ SHARE } \\
\hline Northern EU & 3 & -5122.29 & 10332.25 & $\underline{0.82}$ & $<0.0001$ & $93(5 \%)$ \\
\hline Western EU & 3 & -23131.56 & 46380.23 & $\underline{0.74}$ & $<0.0001$ & $936 \underline{(11 \%)}$ \\
\hline Southern EU & 2 & -11466.84 & 23000.53 & $\underline{0.99}$ & $<0.0001$ & $935(23 \%)$ \\
\hline Eastern EU & 3 & -22092.06 & 44299.40 & $\underline{0.76}$ & $<0.0001$ & $1267(17 \%)$ \\
\hline JSTAR-Japan & 3 & -5475.88 & 11040.55 & $\underline{0.84}$ & 0.02 & $322(16 \%)$ \\
\hline KLoSA-Korea & 2 & -9472.86 & 19010.16 & $\underline{0.70}$ & $<0.0001$ & $1366(40 \%)$ \\
\hline CHARLS-China & 2 & -17674.40 & 35422.05 & $\underline{0.73}$ & $<0.0001$ & $2676(40 \%)$ \\
\hline \multicolumn{7}{|c|}{ Women } \\
\hline ELSA-UK & 3 & -13462.08 & 27030.84 & $\underline{0.77}$ & $<0.001$ & $1230(25 \%)$ \\
\hline HRS-US & 3 & -33847.29 & 67881.68 & $\underline{0.70}$ & $<0.0001$ & $3118(27 \%)$ \\
\hline \multicolumn{7}{|l|}{ SHARE } \\
\hline Northern EU & 3 & -5820.25 & 11731.24 & $\underline{0.70}$ & $<0.0001$ & $156(7 \%)$ \\
\hline Western EU & 2 & -27002.45 & 54083.63 & $\underline{0.86}$ & $<0.0001$ & $1732(17 \%)$ \\
\hline Southern EU & 2 & -12882.32 & 25834.01 & $\underline{0.79}$ & $<0.0001$ & $2150(43 \%)$ \\
\hline Eastern EU & 3 & -27288.15 & 54697.15 & $\underline{0.80}$ & $<0.0001$ & $2456(24 \%)$ \\
\hline JSTAR-Japan & 2 & -4022.47 & 8103.17 & $\underline{0.94}$ & 0.005 & $352(17 \%)$ \\
\hline KLoSA-Korea & 3 & -9179.08 & 18462.89 & $\underline{0.73}$ & 0.05 & $316(7 \%)$ \\
\hline CHARLS-China & 1 & -12625.05 & 25303.08 & 1 & / & $6843(100 \%)$ \\
\hline
\end{tabular}

$†$ Selected cluster solution was model with a lower aBIC (adjusted Bayesian Information criterion), a higher entropy (closer to one), p-value of LMR-LRT (Lo-Mendell Rubin likelihood ratio test) $<0.05$ (i.e. models with additional cluster improved the model 
fit) and cluster size no less than $5 \%$ of the study sample.

10 


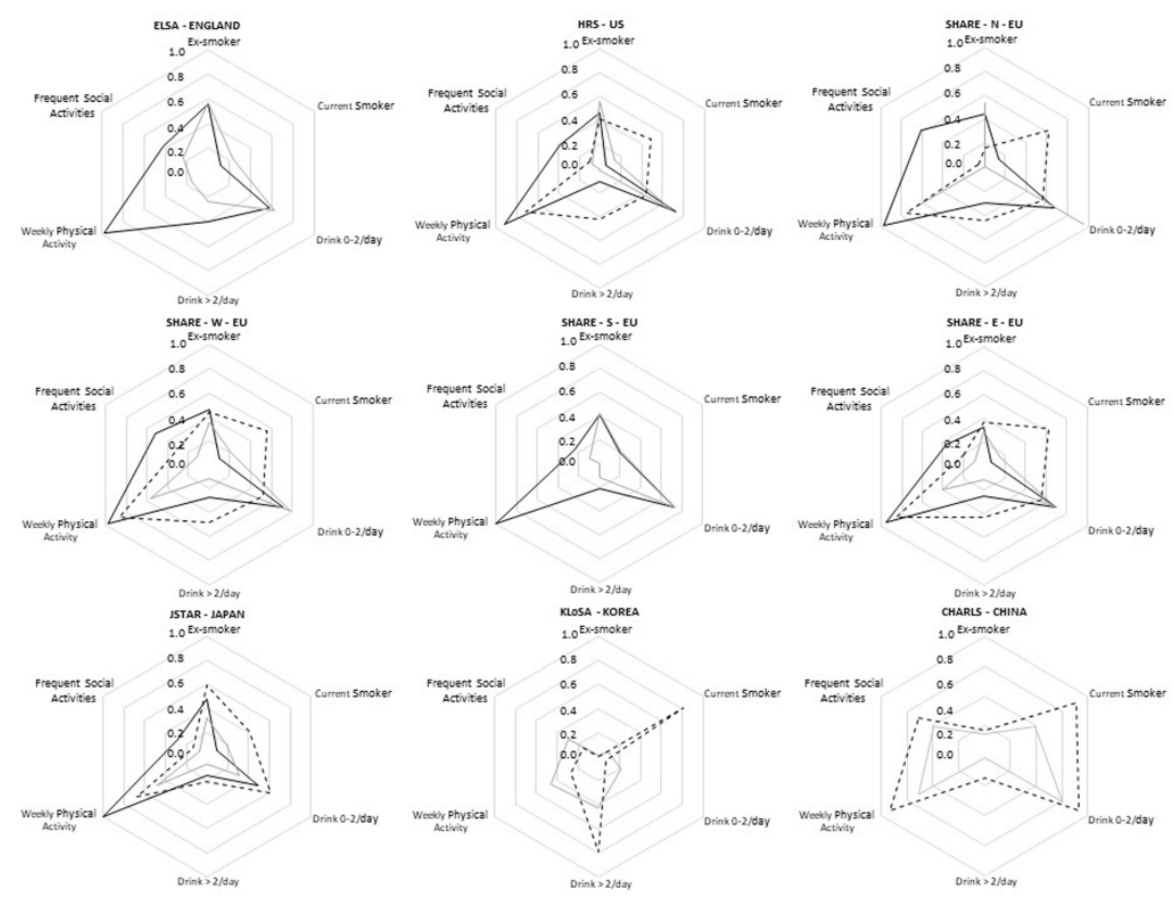

Figure 1. Radar charts of item-response probabilities of health-related behavior clustering for three latent clusters by cohort in men. 'Multiple-HRBs': ex-smoking, moderate drinking, frequent physical and social activity (Black line); 'Inactives': socially and physically inactive without other risk behaviors (Grey line); and '(ex)Smokers with Risk Behaviors' (Black dash line). Data from participants aged $\geq 50$ years in 2010 Health and Retirement Study (HRS), English Longitudinal Study of Ageing (ELSA), the Survey of Health Ageing and Retirement in Europe (SHARE), Japanese longitudinal study of Ageing (JSTAR), Korean Longitudinal Study of Aging (KLOSA) and China Health and Retirement Longitudinal Study (CHARLS).

$275 \times 190 \mathrm{~mm}(300 \times 300 \mathrm{DPI})$ 


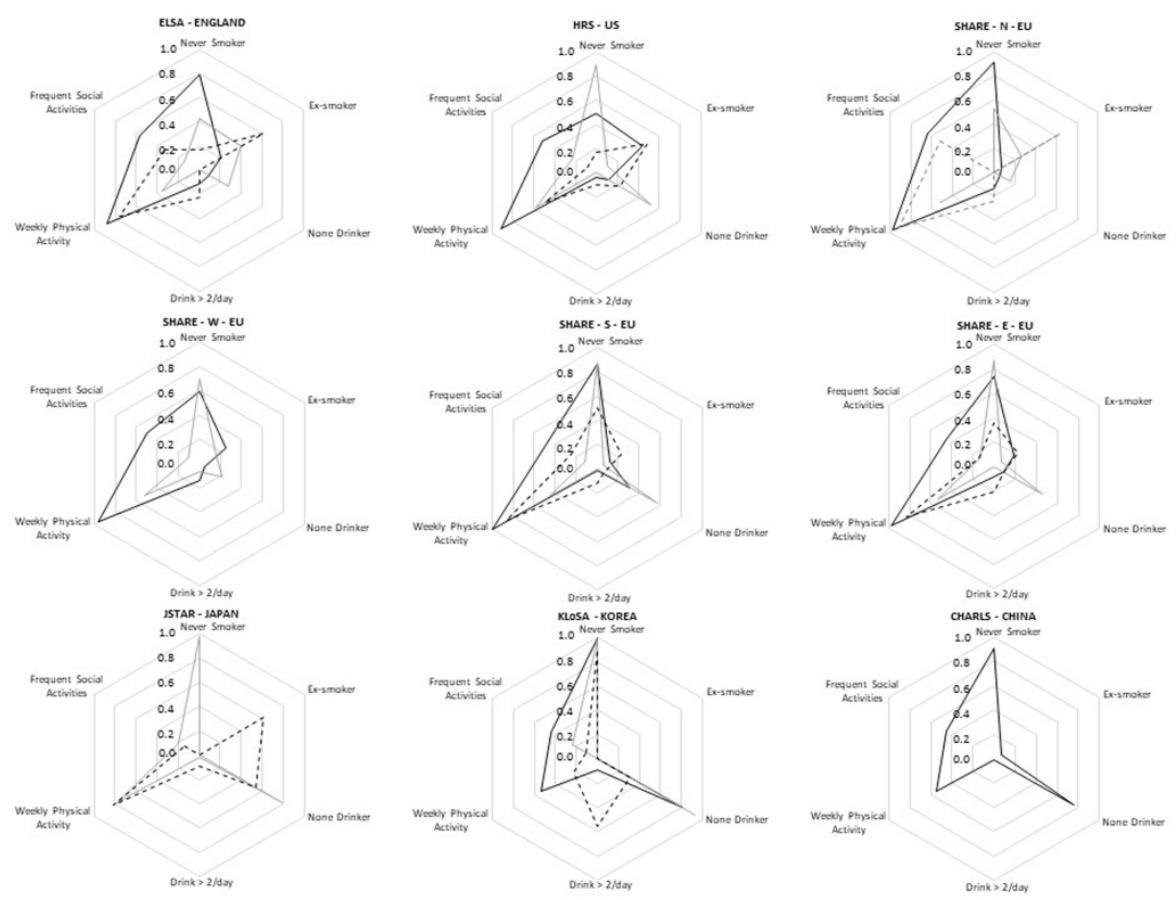

Figure 2. Radar charts of item-response probabilities of health-related behavior clustering for three latent clusters by cohort in women. 'Multiple-HRBs': never-smoking, non/moderate drinking, frequent physical and social activity (Black line); 'Inactives': socially and physically inactive without other risk behaviors (Grey line); and '(ex)Smokers with Risk Behaviors' (Black dash line). Data from participants aged $\geq 50$ years in 2010 Health and Retirement Study (HRS), English Longitudinal Study of Ageing (ELSA), the Survey of Health Ageing and Retirement in Europe (SHARE), Japanese longitudinal study of Ageing (JSTAR), Korean Longitudinal Study of Aging (KLOSA) and China Health and Retirement Longitudinal Study (CHARLS). $275 \times 190 \mathrm{~mm}(300 \times 300 \mathrm{DPI})$ 


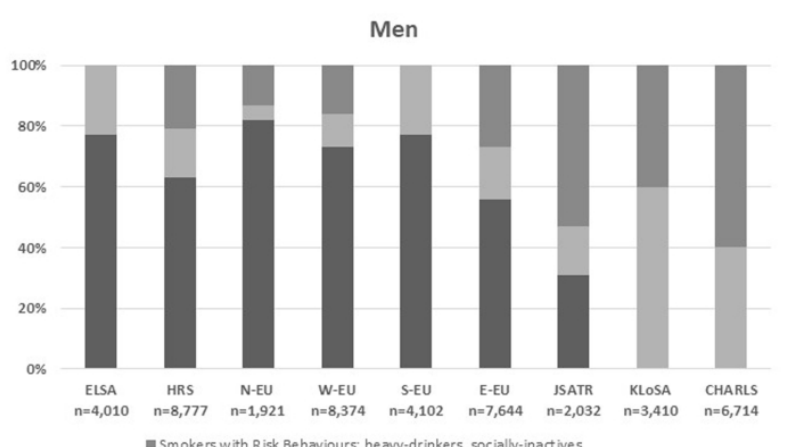

II Inactives: Socially \& physically inactive, ex-smokers \& moderate-drinkers

- Multiple-HRBs: Ex-smokers, moderate-drinkers, physically \& socially active

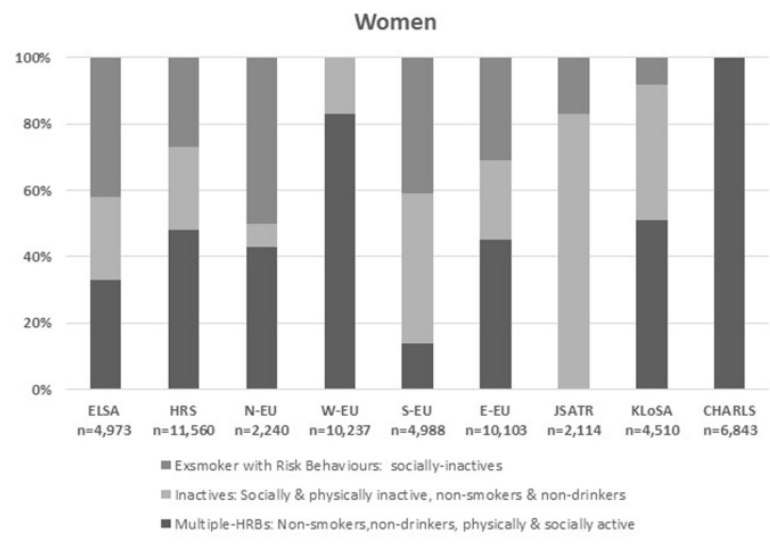

Figure 3. The proportions of health-related behavior cluster membership by gender and cohort. Data from participants aged $\geq 50$ years in 2010 Health and Retirement Study (HRS), English Longitudinal Study of Ageing (ELSA), the Survey of Health Ageing and Retirement in Europe (SHARE), Japanese longitudinal study of Ageing (JSTAR), Korean Longitudinal Study of Aging (KLoSA) and China Health and Retirement Longitudinal Study (CHARLS).

$$
190 \times 338 \mathrm{~mm}(300 \times 300 \text { DPI })
$$

